\title{
Dual capacitive-inductive nature of periodic graphene patches: Transmission characteristics at low-terahertz frequencies
}

\author{
Yashwanth R. Padooru, ${ }^{1}$ Alexander B. Yakovlev, ${ }^{1}$ Chandra S. R. Kaipa, ${ }^{1,}$ George W. Hanson, ${ }^{2}$ \\ Francisco Medina, ${ }^{3}$ and Francisco Mesa ${ }^{4}$ \\ ${ }^{1}$ Center for Applied Electromagnetic Systems Research (CAESR), Department of Electrical Engineering, University of Mississippi, \\ University, Mississippi 38677-1848, USA \\ ${ }^{2}$ Department of Electrical Engineering, University of Wisconsin-Milwaukee, 3200 N. Cramer St., Milwaukee, Wisconsin 53211, USA \\ ${ }^{3}$ Department of Electronics and Electromagnetism, University of Seville, Seville 41012, Spain \\ ${ }^{4}$ Department of Applied Physics 1, University of Seville, Seville 41012, Spain
}

(Received 21 December 2012; revised manuscript received 13 February 2013; published 4 March 2013)

\begin{abstract}
We report on the dual nature (capacitive and inductive) of the surface impedance of periodic graphene patches at low-terahertz frequencies. The transmission spectra of a graphene-dielectric stack shows that patterned graphene exhibits both the low-frequency (capacitive) passband of metal patch arrays and the higher-frequency (inductive) passband of metal aperture arrays in a single tunable configuration. The analysis is carried out using a transfer-matrix approach with two-sided impedance boundary conditions, and the results are verified using full-wave numerical simulations. In addition, the Bloch-wave analysis of the corresponding infinite periodic structure is presented in order to explain the passband and stopband characteristics of the finite graphene-dielectric stack.
\end{abstract}

DOI: 10.1103/PhysRevB.87.115401

PACS number(s): 78.20.-e, 41.20.Jb, 42.25.Bs, 42.70.Qs

\section{INTRODUCTION}

In recent years, there has been considerable interest in the analysis of electromagnetic transmission through a variety of stacked periodic surfaces due to their broad range of filter applications. With the latest developments in fabrication technology, the transmission and reflection spectra of these structures can be realized at optical, ${ }^{1-4}$ infrared, ${ }^{5,6}$ terahertz, ${ }^{7-9}$ and microwave ${ }^{10-13}$ frequencies through careful design of the constituent subwavelength periodic elements and dielectric layers. For example, these include a stack of metal apertures (mesh-grids) at microwave ${ }^{12}$ and infrared frequencies, ${ }^{5,6}$ a stack of metallic patch arrays at microwave frequencies, ${ }^{13}$ metal-dielectric and aperture/mesh-grid-dielectric stacks at optical frequencies, ${ }^{1-4}$ and more recently a stack of graphene sheets-dielectric layers at low-terahertz frequencies. ${ }^{9}$ Various periodically patterned graphene surfaces ${ }^{14-18}$ have been designed at microwave, terahertz, and optical frequencies for tunable metamaterials, with potential applications including filters, absorbers, and polarizers. Of particular interest is the low-terahertz band which has seen an increase in graphene applications (e.g., cloaking ${ }^{19}$ and plasmonic oscillators, ${ }^{20}$ among others) due to the low real part of its surface conductivity, forming a low-loss surface reactance.

The spectra of these multilayer configurations consist of a series of passband and stopband regions, and the corresponding resonances of transmission within the passband are explained in terms of coupled Fabry-Pérot resonances of the individual reactively loaded dielectric slabs (that are strongly coupled through the patterned metallic surfaces or graphene sheets). Further, it was discussed in Ref. 12 that the stacked metallic apertures/mesh-grids at microwave frequencies mimic the transmission properties of a metal-dielectric stack at optical frequencies, and in Ref. 9 it was shown that a graphene sheetdielectric stack at low-terahertz frequencies behaves similar to metallic apertures/mesh-grids. In general, metal patch structures have a capacitive surface reactance, and exhibit a low-frequency passband followed by alternating stop and (generally poorer quality) passbands as frequency increases [see Fig. 4(c)]. Alternatively, aperture/mesh-grid structures, which are complementary to the metal patch structures in the Babinet sense, ${ }^{21}$ have an inductive surface reactance and exhibit a low-frequency stopband, followed by alternating pass and stopbands as frequency increases [see Fig. 4(d)].

In this work, we continue our study of transmission properties of a graphene-dielectric stack at low-terahertz frequencies, ${ }^{9}$ with the graphene monolayers replaced by twodimensional (2D) periodic graphene patches (with a typical geometry shown in Fig. 1). We demonstrate that because of its relatively long electronic mean-free path, patterned graphene can exhibit both the low-frequency passband/stopband characteristics of capacitive periodic metallic patches and the complementary inductive nature of aperture/mesh-grid arrays (also exhibited by graphene monolayers at low-terahertz frequencies). This functionality is due to the fact that, as explained below and which is the main point of the paper, at low frequencies the graphene patches behave as a capacitive reactive surface similar to metallic patches, and at higher frequencies graphene patches behave as an inductive reactive surface similar to a metallic aperture/mesh-grid array (or a graphene monolayer). The use of graphene in such structures is desirable due to its tunability and unique electrical, thermal, and mechanical properties: high electrical conductivity, optical transparency, and high tensile strength, among others. With developments in the fabrication of large graphene layers using chemical vapor deposition, ${ }^{22}$ graphene has become an attractive candidate for many high-speed electronic and electromagnetic applications, ${ }^{23-34}$ ranging from microwave to optical frequencies.

The analysis in this paper is carried out with a transfermatrix approach for dielectric slabs and two-sided impedance boundary conditions applied at the graphene patch-dielectric 

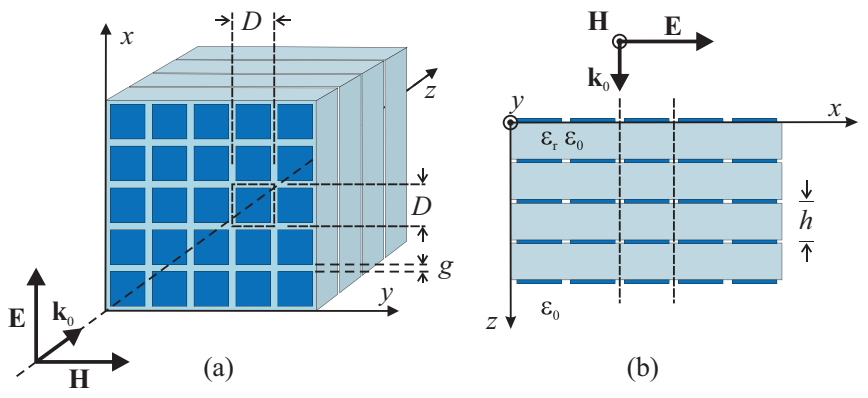

FIG. 1. (Color online) Geometry of a stack of periodic graphene or metal patches separated by dielectric slabs with a plane wave at normal incidence. (a) 3D view and (b) cross-section view.

interfaces. In all numerical results, the full graphene intraband and interband conductivity is used, ${ }^{35,36}$ although the analytical manipulations that show how the capacitive or inductive nature of the surface becomes manifest is based on the Drude intraband conductivity, which is dominant in the low- $\mathrm{THz}$ band of interest. Because of the subwavelength dimensions, the patches are accurately represented by a closed-form analytical surface impedance expression, obtained as a quasidynamic solution of the scattering problem. Moreover, Bloch-wave analysis of the corresponding infinite periodic structure is presented in order to explain the passband and stopband behaviors in the finite graphene-dielectric stack. Assuming a Drude response, the analytical results are validated against full-wave numerical simulations [high frequency structure simulator (HFSS).$^{37}$

The paper is organized as follows. In Sec. II, we present the analytical model for the surface impedance of patterned graphene or metal, demonstrating the capacitive nature of metallic patches but the possible dual nature (capacitive and inductive) of graphene patches (or any long mean-free-path material). In Sec. III, we provide a detailed description of the transmission behavior through the stack of graphene patches, and compare with metal patches. Further, we model the band diagrams of the corresponding infinite structure in order to explain the passband and stopband properties of the finite graphene-dielectric stack. Finally, conclusions are drawn in Sec. IV. A time dependence of the form $e^{j \omega t}$ is assumed and suppressed throughout this study.

\section{THEORETICAL FORMULATION}

A plane wave with the electric field oriented along the $x$ direction is incident normally on a multilayer stack of patch arrays (metal or graphene) with period $D$ and gap $g$ between the patches, separated by dielectric slabs with relative permittivity $\varepsilon_{r}$ and thickness $h$, as shown in Fig. 1 . It is assumed that the parameters of the patch arrays and those of the dielectric slabs in each layer are the same. To understand the difference between having graphene patches and metallic patches, in the following we will consider the patches to be a general material characterized by a complex surface conductivity $\sigma(\mathrm{S})$ having the Drude form $\sigma=\sigma_{0} /(1+j \omega \tau)$, where $\omega$ is the radian frequency and $\tau$ is the phenomenological scattering time. Both graphene and metals can be modeled in this way in the low-THz regime, below the range of interband transitions, and, in fact, the analysis is valid for any Drude material. As will be shown, the value of $\tau$ for a given material is critical in determining if the resulting surface impedance of a patch array is always capacitive, or if it can be inductive. In the latter case, the ratio $\sigma_{0} / \tau$ determines the capacitive or inductive nature of the array. The main focus is on the dual nature of graphene patches in the low $\mathrm{THz}$, but we show that similar behavior occurs for metal patches in the visible spectrum.

For any Drude material we can write the Drude weight as

$$
\sigma_{0}=\alpha \tau,
$$

where, for a metal sheet of thickness $d$,

$$
\alpha^{\mathrm{m}}=\frac{e^{2} n_{e}}{m_{e}} d
$$

where $-e$ is the charge of an electron, $n_{e}$ is the electron density $\left(\mathrm{m}^{-3}\right)$, and $m_{e}$ is the electron mass [here we write the two-dimensional (surface) conductivity as the product of the three-dimensional conductivity and the metal thickness]. For graphene, ${ }^{35,36}$

$$
\alpha^{\mathrm{g}}=\frac{e^{2} k_{B} T}{\pi \hbar^{2}}\left\{\frac{\mu_{c}}{k_{B} T}+2 \ln \left(e^{-\frac{\mu_{c}}{k_{B} T}}+1\right)\right\},
$$

where $\hbar=h / 2 \pi$ is the reduced Planck's constant, $k_{B}$ is Boltzmann's constant, $T$ is temperature, and $\mu_{c}$ is the chemical potential.

Assuming typical values of $\tau$, the surface conductivity of graphene in the low- $\mathrm{THz}$ range is complex valued having a negative imaginary part and a small real part, so that the resulting surface impedance is inductive. As an example, at $1 \mathrm{THz}, T=300 \mathrm{~K}$, and $\mu_{c}=0, \sigma^{\mathrm{g}}=0.194-j 0.609 \mathrm{mS}$ using $\tau^{\mathrm{g}}=0.5 \mathrm{ps}$ (corresponding to a mean-free path of several hundred nanometers). This value of the scattering time for graphene is similar to that measured in Refs. 38 (1.1 ps), 39 $(0.35 \mathrm{ps})$, and $40(0.33 \mathrm{ps})$, where a Drude conductivity was verified in the far infrared. The resulting surface impedance is $Z_{s}^{\mathrm{g}}=1 / \sigma^{\mathrm{g}}=474.3+j 1490.1 \Omega$. For metal at the same temperature and frequency, $\sigma^{\mathrm{m}}=(32.7-j 4.1) d \mathrm{mS}$, where $d$ is measured in nm, assuming typical parameters $n_{e}=5.9 \times$ $10^{28} \mathrm{~m}^{-3}$ and $\tau^{\mathrm{m}}=20 \mathrm{fs}$ (this value would be smaller for thin metal sheets due to surface and grain-boundary scattering ${ }^{41}$ ), such that $Z_{s}^{\mathrm{m}}=1 / \sigma^{\mathrm{m}}=(30.1+j 3.8) / d \Omega$. Therefore, for monolayers both graphene and metal have an inductive surface impedance, with the graphene being much more inductive than the metal. Physically, this is due to the relatively long electron mean-free path in graphene, resulting in a large kinetic inductance.

For a patch array as depicted in Fig. 1, since the dimensions of the unit cell are assumed to be subwavelength, the patch array surface in each layer can be characterized by a homogeneous surface impedance $Z_{s},{ }^{42-45}$

$$
\begin{aligned}
Z_{s} & =Z_{s 1}+Z_{s 2} \\
& =\frac{D}{(D-g) \sigma}-j \frac{\pi}{2 \omega \varepsilon_{0} \varepsilon_{r}^{q s} D \ln \left\{\csc \left(\frac{\pi g}{2 D}\right)\right\}},
\end{aligned}
$$

where $D$ is the period, $g$ is the gap size, $\varepsilon_{r}^{q s}=\varepsilon_{r}$ for interior layers and $\varepsilon_{r}^{q s}=\left(\varepsilon_{r}+1\right) / 2$ for layers at the top and bottom interfaces. One can represent the above impedance as a series $R-L-C$ circuit $Z_{s 1}+Z_{s 2}$, where the first impedance 
corresponds to the series $R-L$ given by the product of the resistive-inductive surface impedance $Z_{s}=1 / \sigma$ and the geometric factor $D /(D-g)$. The second impedance $Z_{s 2}$ corresponds to $-j /\left(\omega C_{\text {eff }}\right)$, a capacitive impedance associated with the patch geometry and background environment, where $C_{\text {eff }}=(2 / \pi) \varepsilon_{0} \varepsilon_{r}^{q s} D \ln \{\csc [\pi g /(2 D)]\}$. Inserting the Drude conductivity into the expression for surface impedance (4) leads to

$$
Z_{s}=\frac{D}{(D-g)} \frac{1+j \omega \tau}{\sigma_{0}}-j \frac{1}{\omega C_{\mathrm{eff}}} .
$$

At this point, we distinguish between effective $(\omega \tau \ll 1)$ and ineffective $(\omega \tau \gg 1)$ electron scattering regimes in the low $\mathrm{THz}$, assuming the typical parameters $\tau^{\mathrm{m}}=20$ fs and $\tau^{\mathrm{g}}=$ $0.5 \mathrm{ps}$. The different physical behavior of these two materials is due to the fact that $\tau^{\mathrm{m}} \ll \tau^{\mathrm{g}}$. If electron scattering is effective, $\omega \tau \ll 1$, which is not true for graphene in the low $\mathrm{THz}$ assuming its typical long mean-free path but which is satisfied in this frequency range by typical metals at room temperature (e.g., at $1 \mathrm{THz}, \omega \tau^{\mathrm{m}} \simeq 0.13$; even smaller $\tau^{\mathrm{m}}$ values will likely result due to enhanced surface and grain-boundary scattering in thin metal sheets, ${ }^{41}$ which would even more forcefully satisfy the inequality), then

$$
Z_{s}^{(\omega \tau \ll 1)}=\frac{D}{(D-g)} \frac{1}{\sigma_{0}}-j \frac{1}{\omega C_{\text {eff }}},
$$

and the surface impedance has real resistance and capacitive reactance. In this case, the geometric patch capacitance dominates the (weakly) inductive nature of the material. This always occurs for metal patches in the low THz. However, if $\omega \tau \gg 1$, which is not true for typical metals at room temperature but which is approximately true for graphene (e.g., at $1 \mathrm{THz}, \omega \tau^{\mathrm{g}} \simeq 3.1$ ), then

$$
Z_{s}^{(\omega \tau \gg 1)}=j\left(\frac{D}{(D-g)} \frac{\omega \tau}{\sigma_{0}}-\frac{1}{\omega C_{\text {eff }}}\right)
$$

and grid impedance is a pure reactance: positive (i.e., inductive) if $\alpha<\beta$ and negative (i.e., capacitive) if $\alpha>\beta$, where $\alpha=\sigma_{0} / \tau$ and

$$
\begin{aligned}
\beta & =\frac{2}{\pi} \omega^{2} \varepsilon_{0} \varepsilon_{r}^{q s} D \ln \left[\csc \left(\frac{\pi g}{2 D}\right)\right] \frac{D}{(D-g)} \\
& =\omega^{2} C_{\text {eff }} \frac{D}{(D-g)} .
\end{aligned}
$$

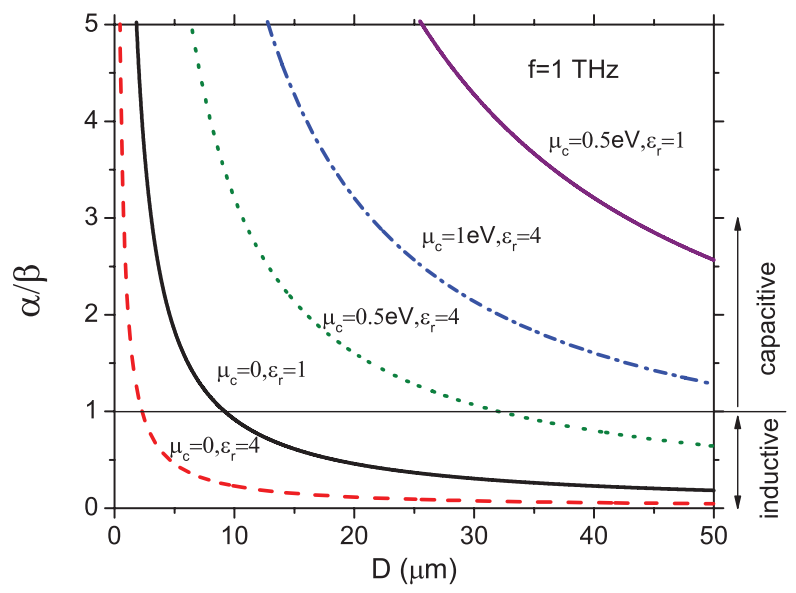

(a)

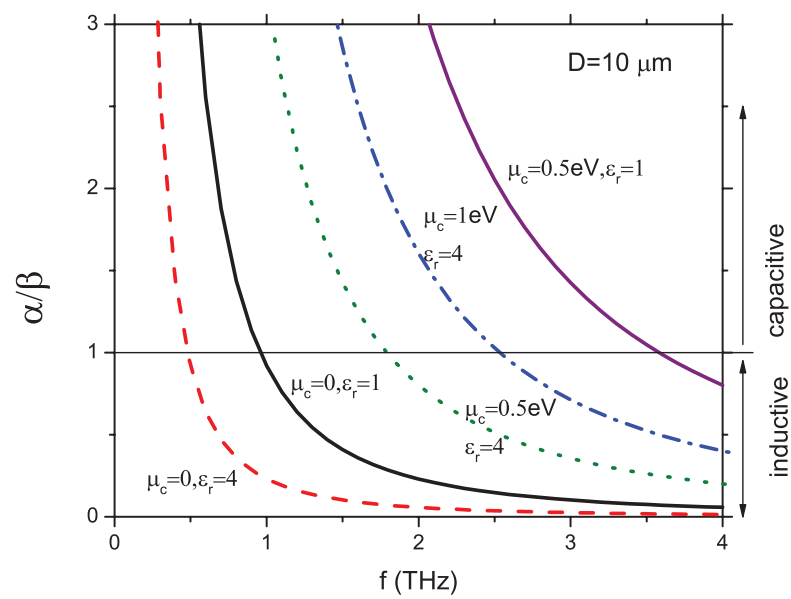

(c)

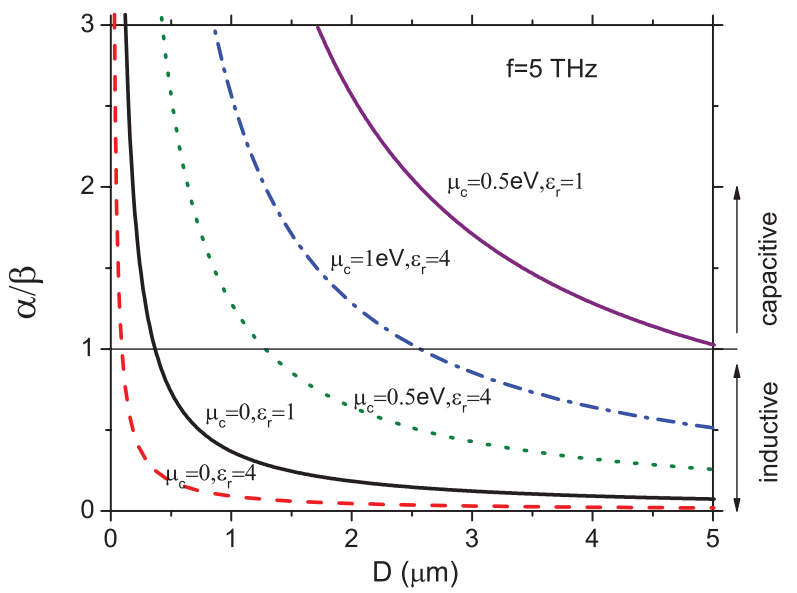

(b)

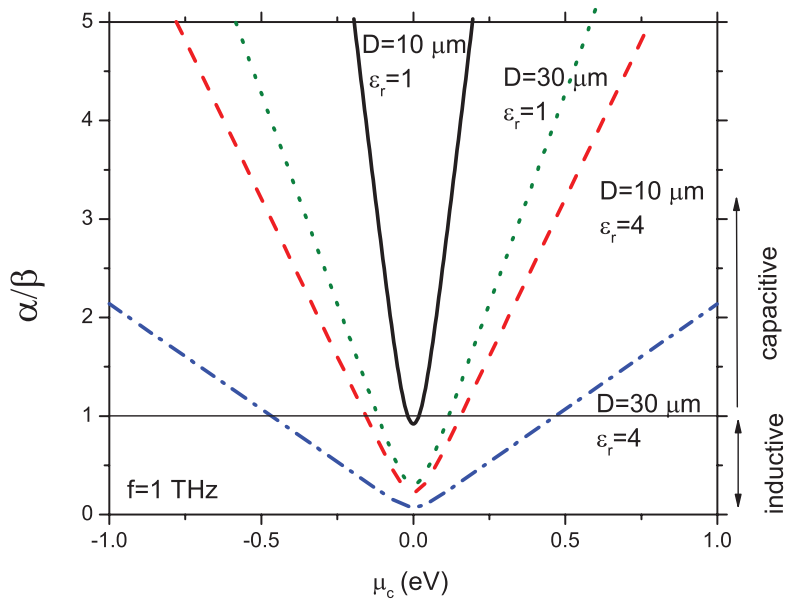

(d)

FIG. 2. (Color online) (a), (b) The ratio $\alpha / \beta$ is shown for graphene patches at 1 and $5 \mathrm{THz}$ vs period $D$ for various combinations of chemical potential and permittivity. (c) The ratio $\alpha / \beta$ for a fixed period $D=10 \mu \mathrm{m}$ as a function of frequency. (d) The ratio $\alpha / \beta$ as a function of chemical potential $\mu_{c}$ for various combinations of period $D$ and permittivity calculated at $1 \mathrm{THz}$. In all the calculations, the gap between the patches $g=D / 10$. 
In the inductive case, the geometric patch capacitance does not dominate the (strong) inductive nature of the material. This inductive behavior can be observed even for $(\omega \tau \gtrsim 1)$, in which case the surface impedance is not a pure reactance but is complex valued with an inductive reactance. In summary, for a material such as graphene with a sufficiently long mean-free path, we can achieve either an inductive or capacitive surface reactance of the planar patch array by adjusting the normalized Drude weight $\alpha$.

In Figs. 2(a) and 2(b), the ratio $\alpha / \beta$ is shown for graphene patches at 1 and $5 \mathrm{THz}$ versus period $D$ for various combinations of $\mu_{c}$ and $\varepsilon_{r}$ (other numerical parameters are the same as described above). It can be seen that for small periods we have $\alpha>\beta$, so that the resulting surface reactance is capacitive, as occurs for metal patches. Above a critical period $D$ at which $\alpha=\beta$ we obtain $\alpha<\beta$ and the surface reactance becomes inductive. Figure 2(c) shows the ratio $\alpha / \beta$ for a fixed period $D=10 \mu \mathrm{m}$ as a function of frequency; at low frequencies, the surface reactance is capacitive and becomes inductive above a critical frequency where $\alpha=\beta$. In Fig. 2(d), we depict the ratio $\alpha / \beta$ calculated at the frequency of $1 \mathrm{THz}$ as a function of $\mu_{c}$ for various combinations of period $D$ and $\epsilon_{r}$. It can be observed that for a given period and permittivity, the reactance can be tuned via the chemical potential to be inductive or capacitive.

In Fig. 3, the imaginary part of the surface impedance (4) as a function of frequency is shown for a single layer of free-standing graphene and metallic patches, as well as, for reference, the case of contiguous graphene and metallic sheets. [Here and in all following results, the full graphene intraband and interband conductivities are used; ${ }^{35,36}$ in the low THz the intraband is the dominant contribution. For the metal results, we set the metal thickness $d$ as the (frequency variable) skin depth.] All cases are for a dielectric host medium having $\varepsilon_{r}=$ 4 , and for the patches $D=10 \mu \mathrm{m}$ and $g=1 \mu \mathrm{m}$. It can be seen that for the contiguous sheets, the graphene and metal sheets always result in an inductive surface reactance (the metallic sheet case is weakly inductive and lies just above the origin of the vertical axis). For the patch arrays, the surface impedance of the metallic patches is always capacitive, whereas the surface impedance of the graphene patches changes from capacitive to inductive as the frequency increases. It can be observed that at low frequencies the behavior of the graphene patches is similar to that of the metallic patches (capacitive), and at high frequencies the behavior of the graphene patches becomes similar to that of a graphene sheet (inductive). This dual property of graphene patches is the motivation to study the transmission properties through the multilayer stack depicted in Fig. 1. Furthermore, the frequency $f_{c}$ (see Fig. 3) at which the transition from capacitive to inductive surface impedance occurs for graphene patches can be electronically tuned via the chemical potential $\mu_{c}$, and also controlled by varying $D$ and $g$.

It would seem that we could also achieve $\omega \tau \gg 1$ with metal patches at low temperature since $\tau$ increases as temperature is lowered. However, it is easy to show that this will still result in only a capacitive reactance (i.e., $\alpha^{\mathrm{m}} / \beta>1$ ). To see this, consider that for a metal the plasma frequency satisfies $\omega_{p}^{2}=e^{2} n_{e} / \varepsilon_{0} m_{e}$, and that we can express $\alpha^{\mathrm{m}}=\omega_{p}^{2} \varepsilon_{0} d$ and

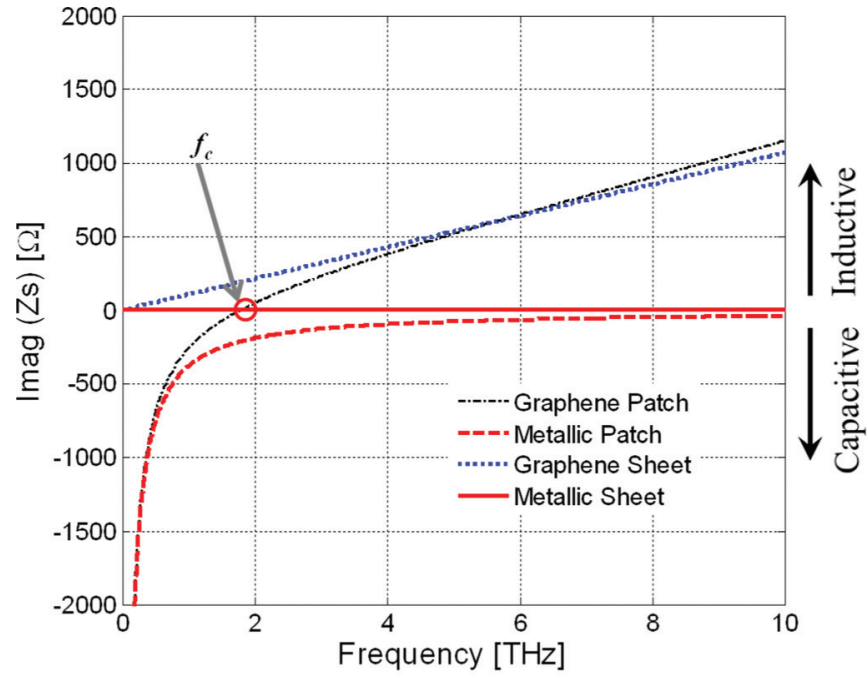

(a)

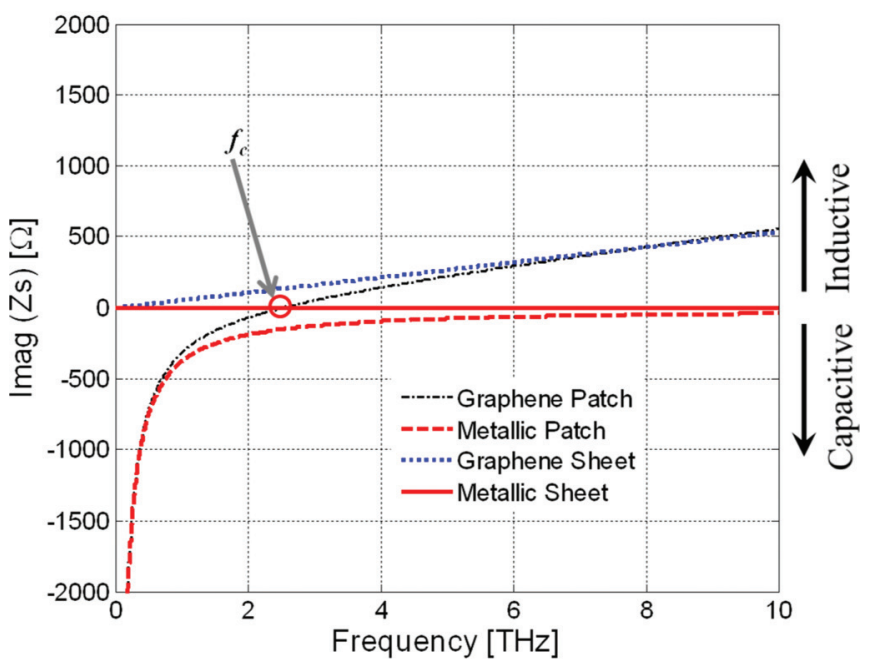

(b)

FIG. 3. (Color online) Imaginary part of the surface impedance $Z_{s}$ of a free-standing graphene patch array, graphene monolayer, metallic patch array, and metallic sheet embedded in a dielectric host of $\epsilon_{r}=4$ : (a) $\mu_{c}=0.5 \mathrm{eV}$ and (b) $\mu_{c}=1 \mathrm{eV}$.

estimate $\beta$ as

$$
\begin{aligned}
\frac{\beta}{\omega^{2} \varepsilon_{0} d} & =\frac{2}{\pi} \varepsilon_{r}^{q s} \frac{D}{d} \ln \left[\csc \left(\frac{\pi g}{2 D}\right)\right] \frac{D}{(D-g)} \\
& \sim \frac{2}{\pi} \varepsilon_{r}^{q s} \frac{D}{d} \sim \frac{D}{d},
\end{aligned}
$$

so that

$$
\alpha^{\mathrm{m}}=\omega_{p}^{2} \varepsilon_{0} d \lesseqgtr \beta \sim \omega^{2} \varepsilon_{0} d \frac{D}{d} .
$$

The inequality $\alpha^{\mathrm{m}} \lesseqgtr \beta$ is then consistent with

$$
\frac{\omega_{p}^{2}}{\omega^{2}} \lesseqgtr \frac{D}{d} .
$$

If we assume typical periods in the $\mu \mathrm{m}$ range and typical metal thickness values in the $\mathrm{nm}$ to $\mu \mathrm{m}$ range (typical skin 
depth values are in this range), then $D / d \sim 1-100$, and so

$$
\frac{\omega_{p}}{\omega} \lesseqgtr \delta,
$$

where $\delta$ is a number of order $1-10$, and since $\omega \ll \omega_{p}$ in the $\mathrm{THz}$ range, the grid reactance of a metal patch array will be capacitive even if we achieve $\omega \tau \gg 1$ by decreasing temperature or increasing material purity. In the vicinity of the plasma frequency, an inductive response is possible, as we discuss later.

In the next section, we consider the transmission problem of the multilayer structure (Fig. 1) obtained by the transfer-matrix approach for the dielectric slabs along with the two-sided impedance boundary conditions at the patch-dielectric interfaces, where the properties of the patch surfaces are described by Eq. (4). ${ }^{4-45}$ If the surface impedance expression was exact and the structure was laterally infinite, the transfer-matrix method would yield the exact solution of Maxwell's equations. The results obtained using the above analytical model are validated using full-wave finite-element-based numerical simulations (HFSS), ${ }^{37}$ in which case a surface impedance for the patch array is not specified and the actual patch geometry is modeled (both methods use the same intraband and interband graphene conductivity models). Alternatively, one can use the circuit model described in Refs. 12,13 , and 46 to analyze the

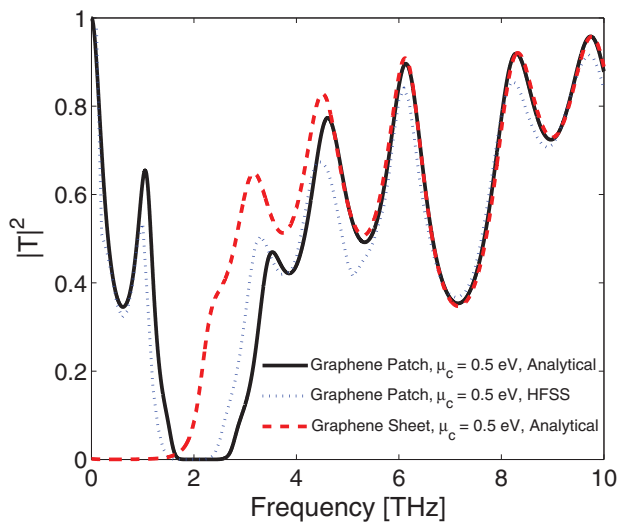

(a)

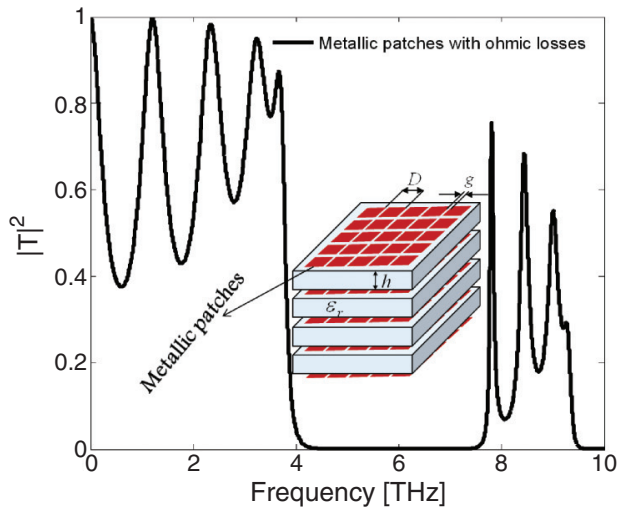

(c) multilayer stack with the graphene patches modeled as a shunt admittance $\left(Y_{s}=1 / Z_{s}\right)$ across the transmission-line sections.

\section{RESULTS AND DISCUSSIONS}

In this section, we study in detail the transmission through a multilayer stack of patches separated by dielectric slabs for a plane wave at normal incidence. We first consider a five-layer stack of graphene patch arrays with period $D=$ $10 \mu \mathrm{m}$ and gap $g=1 \mu \mathrm{m}$, separated by four dielectric slabs each with permittivity $\varepsilon_{r}=4$ and thickness $h=10 \mu \mathrm{m}$. The analytical (matrix-based) results for the transmissivity $|T|^{2}$ through the stack of graphene patches for different values of $\mu_{c}$, along with the transmission properties of metallic patch arrays and metallic apertures/mesh-grids (fishnets), are shown in Fig. 4. Here, similar geometrical parameters are assumed for all four structures. From Figs. 4(a) and 4(b) it can be observed that at low frequencies, the graphene patch arrays (shown using the solid black lines) correspond to a capacitive surface impedance, similar to the metallic patch arrays shown in Fig. 4(c), and, consequently, the transmissivity behavior shows a passband starting from zero frequency and up to a certain upper frequency. As frequency increases, the surface reactance becomes inductive (see Fig. 3) and the transmissivity behavior becomes equal to that of the (inductive, see Fig. 3) graphene sheets (dashed red curves). This is similar to the case of an

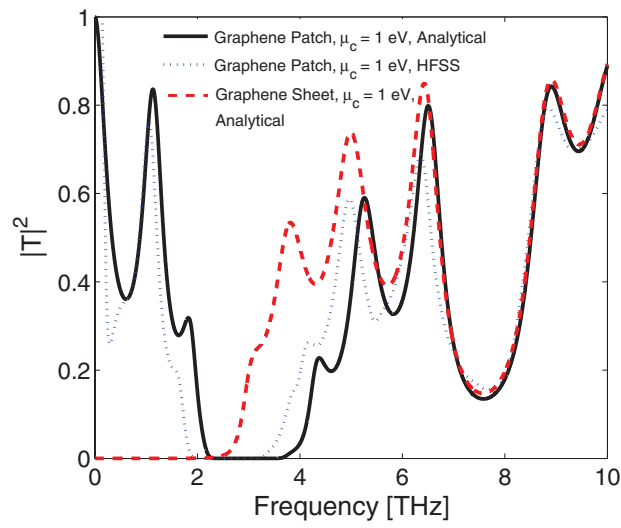

(b)

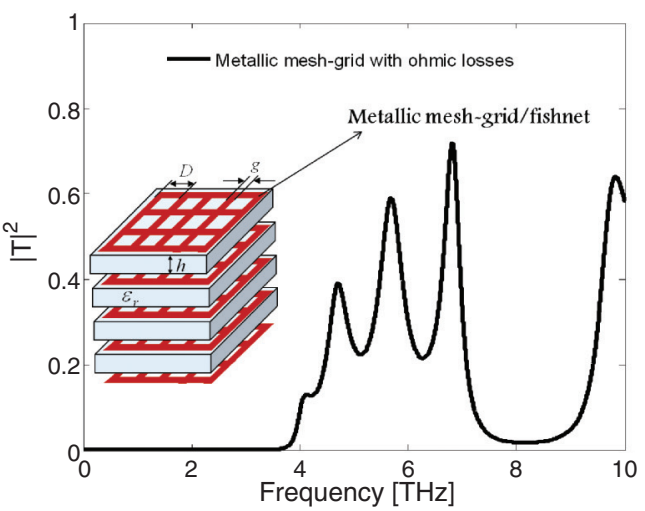

(d)

FIG. 4. (Color online) Transmissivity of different four-layer dielectric stack structures: (a) comparison of stack of graphene patches and graphene sheets for $\mu_{c}=0.5 \mathrm{eV}$, (b) comparison of stack of graphene patches and graphene sheets for $\mu_{c}=1 \mathrm{eV}$, (c) stack of (capacitive) metallic patches, and (d) stack of (inductive) metallic apertures/mesh-grid structures. The HFSS (dotted curves) and transfer-matrix (solid curves) results in (a) and (b) show good agreement. 


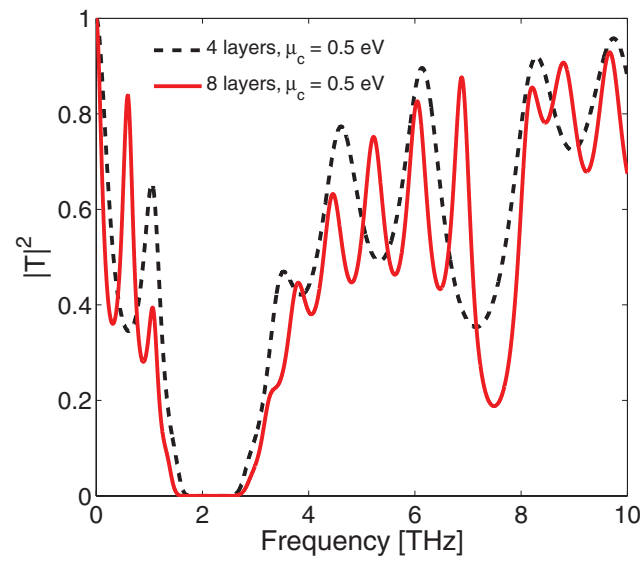

(a)

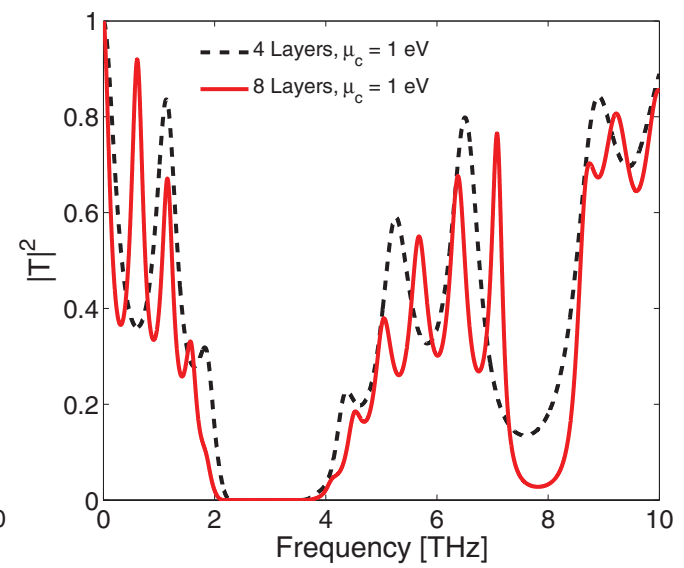

(b)

FIG. 5. (Color online) Analytical results of the transmissivity of a four- and eight-layer graphene patch-dielectric stack: (a) $\mu_{c}=0.5 \mathrm{eV}$ and (b) $\mu_{c}=1 \mathrm{eV}$.

array of apertures/mesh-grids (which are complementary to the metal patch case, and so have a complementary, i.e., inductive, nature), as shown in Fig. 4(d). Clearly, the graphene patch arrays exhibit a combined effect similar to the transmission properties of (capacitive) metal patches at low frequencies and to the (inductive) aperture arrays or solid graphene sheets at higher frequencies. However, the transmission resonances in the first passband are not exactly the same as those of the metallic patches, particularly, the number of transmission resonances, due to the presence of losses in the graphene patches. Nevertheless, these transmission resonances are associated with the Fabry-Pérot resonances of the dielectric slabs loaded with the graphene patches. In addition, in Figs. 4(a) and 4(b) we also show comparisons with full-wave commercial simulations (dotted blue lines) to validate the simple analytical results based on surface impedance (4).

To further show this combined filtering property, in Fig. 5 we plot the transmissivity $|T|^{2}$ for a larger number of layers. One can still clearly notice the low passband (starting from zero frequency), followed by a deep stopband, and then a second passband. Also, it can be noticed that with an increase in the number of layers, the number of transmission peaks which corresponds to the number of coupled layers increases, still maintaining the same characteristic frequency bands.

In order to understand the propagation of electromagnetic waves through the multilayer stack of graphene patches, i.e., to predict the frequency bands of transmission and rejection, here we employ Bloch-wave analysis, similar to that reported in Refs. 12,13, and 47. Following Ref. 47, the dispersion equation for the Bloch waves can be written as

$$
\cos \left(k_{b} h\right)=\cos (\theta)+j \frac{Z_{d}}{2 Z_{s}} \sin (\theta),
$$

where $k_{b}$ is the Bloch wave number, $\theta=k_{d} h$ is the electrical thickness of the slab, $k_{d}=k_{0} \sqrt{\varepsilon_{r}}, k_{0}=\omega / c$ ( $c$ is the velocity of light in vacuum), $Z_{d}=\eta_{0} / \sqrt{\varepsilon_{r}}, \eta_{0}=\sqrt{\mu_{0} / \varepsilon_{0}}$ is the freespace impedance, and $\varepsilon_{r}$ is the permittivity of the dielectric slabs.

The Bloch wave number is real in the region of those frequencies for which $\left|\cos (\theta)+j\left(Z_{d} / 2 Z_{s}\right) \sin (\theta)\right|<1$, which corresponds to propagating waves (passband), and imaginary for complementary (band-gap) regions. The Brillouin diagram for any band can be obtained from Eq. (13). Figure 6 shows the

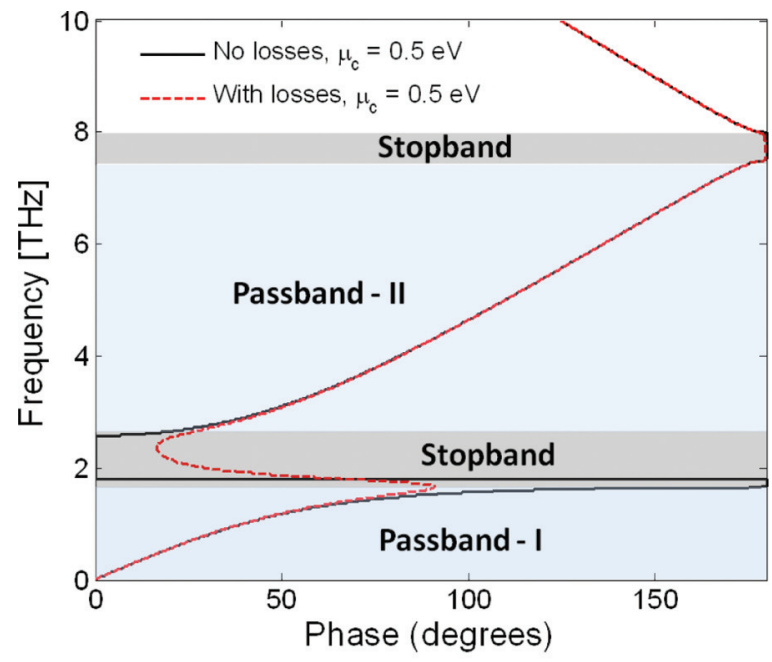

(a)

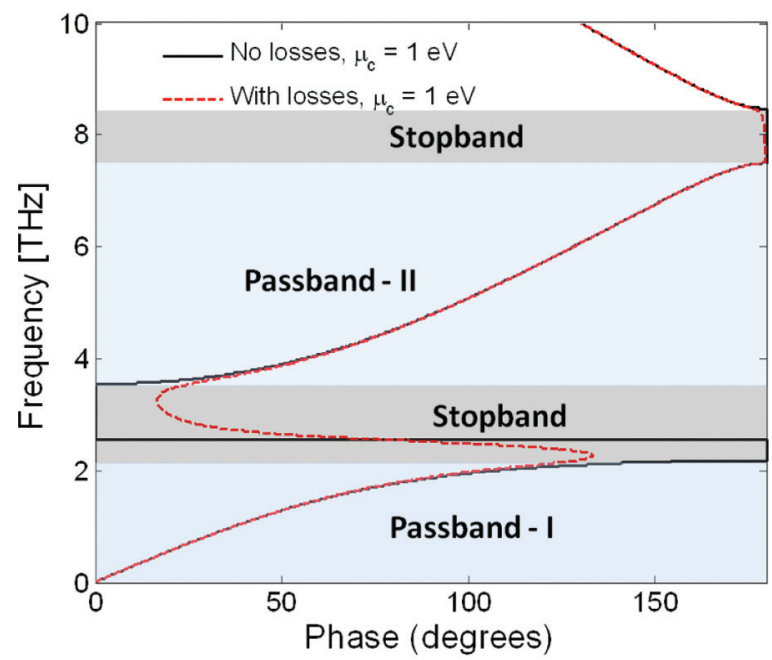

(b)

FIG. 6. (Color online) Brillouin diagram for the transmission bands of an infinite periodic structure with the same unit cell as that used in the results shown in Fig. 5: (a) $\mu_{c}=0.5 \mathrm{eV}$ and (b) $\mu_{c}=1 \mathrm{eV}$. 
band diagrams for the structure under study for two different values of $\mu_{c}(0.5$ and $1 \mathrm{eV})$, with and without ohmic losses in the graphene patch. In the presence of losses one can clearly notice the perturbation in the band behavior. Also, it can be observed that the Brillouin diagrams perfectly predict the passband and stopband regions of the corresponding finite-layer structures [shown in Figs. 5(a) and 5(b)]. The interesting observation is the reversal in the passband regions. That is, after the first stopband, the second passband should in general be backward, however, due to the change in the behavior of the graphene patch arrays (i.e., from capacitive to inductive), one can clearly notice the change in the passband regions. In fact, this change inside the first stopband region occurs at the same frequency where the impedance of the graphene patch changes from inductive to capacitive. This frequency of transition corresponds to absorption by the stack; transmission is practically zero and the reflection is very low (results for reflection are omitted).

To further verify the dual nature of graphene patches, in Figs. 7(b)-7(d), we plot the electric-field distributions for the resonance modes corresponding to the frequency points A, B, and D in Fig. 7(a). Figure 7(b) shows the electricfield distributions along the length of the structure in the propagation direction $(z)$ for the mode $\mathrm{A}$ in the first passband

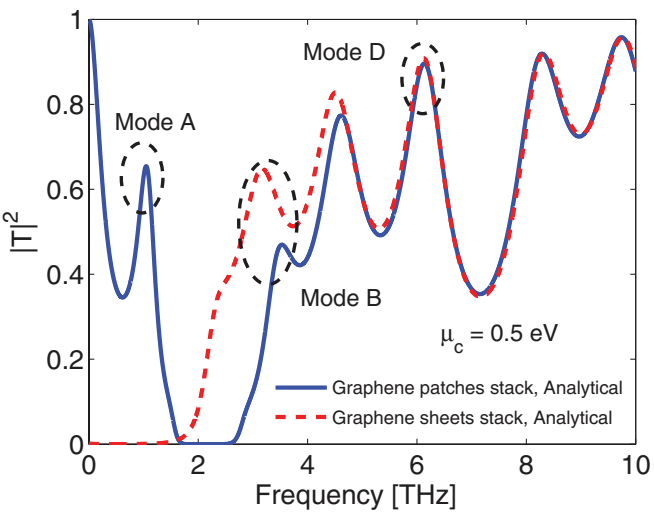

(a)

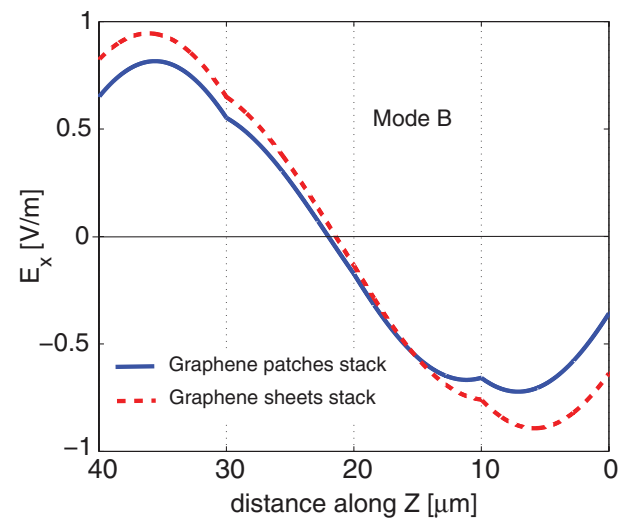

(c) region for two structures: a stack of graphene patch arrays and a stack of metallic patch arrays [the transmissivity $|T|^{2}$ behavior for the stack of metallic patches is not shown here, but can be seen in Fig. 4(c)]. The resonant frequencies of mode $\mathrm{A}$ in the first passband for the two structures are 1.048 and $1.211 \mathrm{THz}$, respectively. Based on the field behavior it is clear that the first passband region of a stack of graphene patches mimics the behavior of stacked metallic patch arrays, however, with some perturbations. Similarly, in Fig. 7(c) we plot the field distributions for another resonance mode of the stacked graphene patches in the second passband region. Since in this region the behavior of graphene patches changes to inductive, it is relevant to compare its field behavior with a similar stack of graphene sheets. Here, we plot the second mode (mode B) with resonant frequencies 3.529 and $3.172 \mathrm{THz}$, respectively. Again, the field behavior of the stack of graphene patches resembles to that of the stack of graphene sheets with some small perturbations in the field. Further, in Fig. 7(d) we show the field behavior of a similar stack of graphene patches and graphene sheets for mode D (with resonant frequencies 6.133 and $6.122 \mathrm{THz}$, respectively) in the second passband region. In this case, based on the transmissivity behavior shown in Fig. 7(a) it is clear that the behavior of the graphene patches is quite similar to that of the graphene sheets. Hence, as

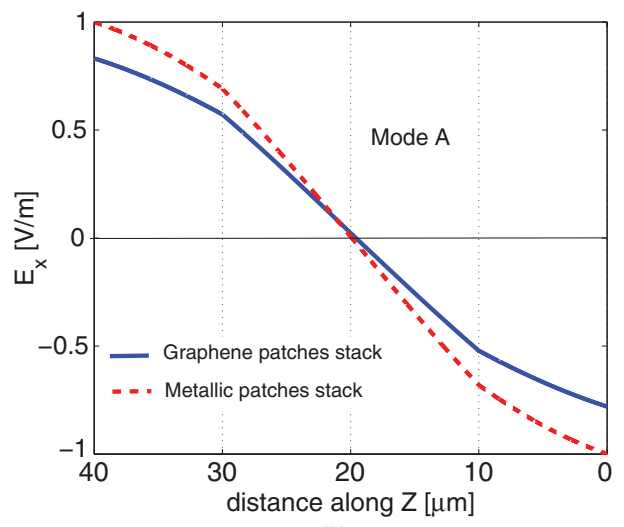

(b)

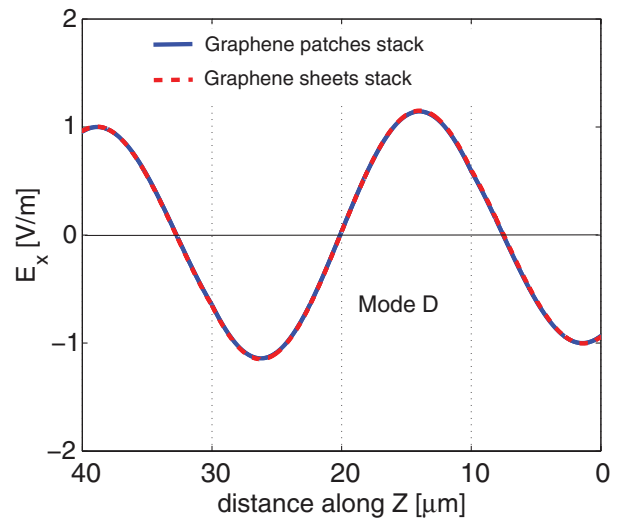

(d)

FIG. 7. (Color online) (a) Comparisons of the transmissivity (calculated using the transfer-matrix approach) for the four-layer graphene patch arrays and graphene sheets-dielectric stack, with $\mu_{c}=0.5 \mathrm{eV}$, (b) comparisons of the electric-field distributions for the resonance mode $\mathrm{A}$ in the first passband of the four-layer graphene patch arrays and metallic patch arrays-dielectric stack, (c) comparisons of the electric-field distributions for the resonance mode B in the second passband of the four-layer graphene patch arrays and graphene sheets-dielectric stack, and (d) comparisons of the electric-field distributions for the resonance mode D in the second passband of the four-layer graphene patch arrays and graphene sheets-dielectric stack. 

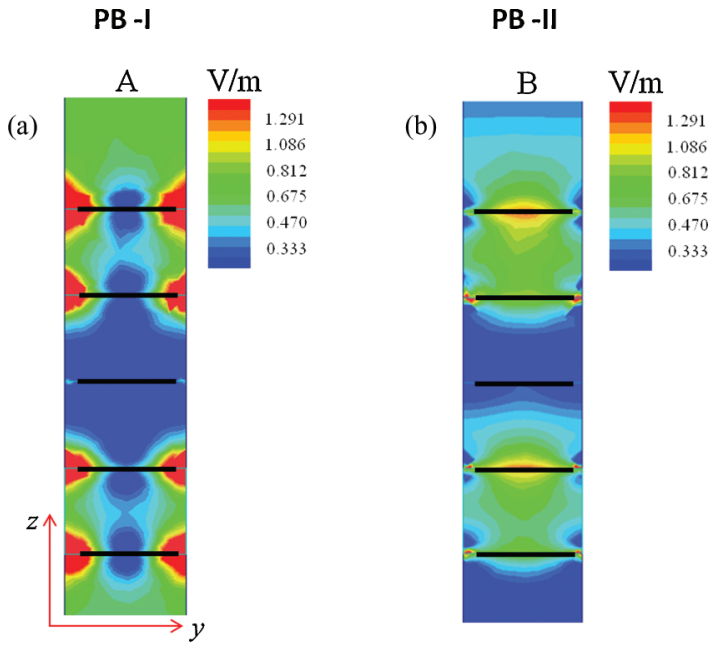

FIG. 8. (Color online) Magnitude of the total electric-field distributions of the resonance modes in the four-layer graphene patch-dielectric stack calculated using HFSS: (a) mode A in the first passband (PB-I) region [corresponding to Fig. 6(b)] and (b) mode B in the second passband (PB-II) region [corresponding to Fig. 6(c)]. The solid black lines represent graphene patches.

expected, the field behaviors shown in Fig. 7(d) are also the same.

In addition, in Fig. 8 we present the magnitude of the total electric-field distributions in the four-layer graphene patchdielectric stack calculated using HFSS, with $\mu_{c}=0.5 \mathrm{eV}$. The results are obtained at the resonant frequencies of the modes A (in first passband region, PB-I) and B (second mode in second passband region, PB-II), clearly demonstrating the field distributions associated with those shown in Figs. 7(b) and 7(c). For mode B [with the field distribution shown in Fig. 8(b)], the field value is low in the middle graphene patch and is concentrated more near the remaining graphene patches, which is consistent with the electric-field distributions shown in Fig. 7(c). Further, this behavior [Fig. 8(b), numerically calculated using HFSS] also resembles to that of the stack of graphene sheets as noticed in Fig. 7(b) in Ref. 9. Similarly, for mode A [with the field distribution shown in Fig. 8(a)], the field value is zero near the middle graphene patch, which is consistent with the electric-field distribution shown in Fig. 7(b). Another interesting fact to be noticed is that in Fig. 8(a), the field behavior of the graphene patch-dielectric stack shows a strong confinement of the field around the edges (which resembles exactly to that of the multilayer metallic patch-dielectric stack) due to a capacitive nature of the patches (results for the stack of metallic patch arrays are not shown here for the sake of brevity). These field behaviors (shown in Fig. 8) clearly demonstrate the dual nature of the graphene patches at low-THz frequencies, i.e., acting as a low-loss capacitive surface at low frequencies, and as a low-loss inductive surface at higher frequencies.

Finally we mention that although as stated above, metal patch arrays always present a capacitive surface reactance in the $\mathrm{THz}$ regime, we observe a dual capacitive-inductive nature of thin metal (silver) patches in the visible spectrum. The Drude model is a poor approximation for metals in this frequency range, and here we use a fit based on measured data $^{48}$ for the permittivity, utilizing an augmented Drude model. ${ }^{49}$ Figure 9(a) shows the surface impedance for three different thickness values $(t)$ of the metal (here $t=d$ is fixed and is not the skin depth as used for the $\mathrm{THz}$ calculations), with the corresponding transmissivity shown in Fig. 9(b) for a five-layer structure, where $D=100 \mathrm{~nm}, g=10 \mathrm{~nm}$, $h=166 \mathrm{~nm}$, and $\epsilon_{r}=1$ (results were computed using the transfer-matrix approach). It can be seen that for the thicker metal case $(t=25 \mathrm{~nm})$, the response is always (nearly) capacitive, as occurs at lower frequencies. For $t=15 \mathrm{~nm}$, there is a range where the surface becomes inductive, and for the thinnest case considered, $t=10 \mathrm{~nm}$, there is a relatively wide frequency range where the patch array acts as an inductive surface. The corresponding transmissivity reflects this, where for the thicker metal the (always capacitive) surface exhibits a low-pass response, but the thinnest metal shows an inductive region starting near $420 \mathrm{THz}$, leading to a second passband. Additionally, as a reference in Fig. 9(b), the transmissivity of the five-layer structure with the metal patch arrays replaced by continuous metal sheets (inductive nature) of same

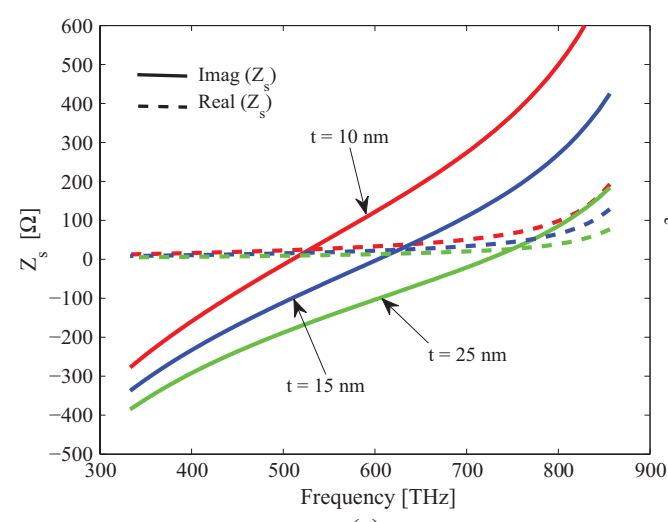

(a)

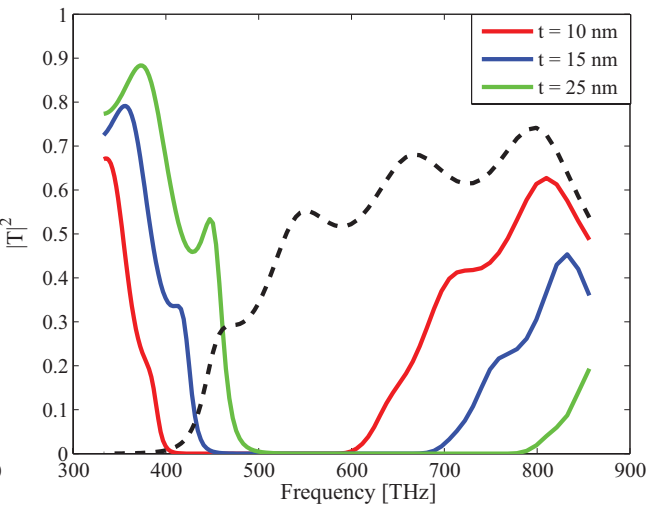

(b)

FIG. 9. (Color online) (a) Surface impedance of metal (silver) patch array for three different thickness values of the metal. The structure is as depicted in Fig. 1, having five layers with $D=100 \mathrm{~nm}, g=10 \mathrm{~nm}, h=166 \mathrm{~nm}$, and $\epsilon_{r}=1$. (b) Transmissivity of the corresponding structure. The dashed black curve corresponds to the transmissivity for the case $t=10 \mathrm{~nm}$, but with the silver patch arrays replaced with the continuous silver film. 
thickness $(t=10 \mathrm{~nm}$ ) is depicted (shown by dashed black curves), showing a low-frequency stopband followed by a passband.

\section{CONCLUSION}

Graphene patches have been shown to have a dual (capacitive and inductive) nature at low-terahertz frequencies, which can be interpreted as the combination of the properties of a multilayer stack of metallic patches (capacitive, wherein the geometric patch capacitance dominates over the weak metal inductance), and the properties of a multilayer stack of contiguous graphene sheets (inductive due to large kinetic inductance of the material) in a single configuration of a multilayer stack of graphene patches. The ultimate nature (capacitive or inductive) of the structure depends on the relative strength of the material kinetic inductance compared to the geometric patch capacitance. The analysis is carried out using a simple analytical model based on surface impedance of the patch array, and the results are validated using full-wave numerical simulations. The transmission resonances are identified as Fabry-Pérot resonances of the coupled patch-dielectric cavities. Further, the characteristics of the transmission bands are explained with the help of band diagrams, modeled using a simple analytical model. This bifunctional property of the graphene patches could be useful in the implementation and design of tunable planar filters and metasurface conformal cloaks for dielectric and metallic cylinders. ${ }^{50,51}$

\section{ACKNOWLEDGMENT}

Support by the Spanish Ministry of Science and Innovation (Projects No. TEC2010-16948 and No. Consolider CSD200800066) is acknowledged. *cskaipa@gmail.com

${ }^{1}$ T. W. Ebbesen, H. J. Lezec, H. F. Ghaemi, T. Thio, and P. A. Wolff, Nature (London) 391, 667 (1998).

${ }^{2}$ M. Scalora, M. J. Bloemer, A. S. Pethel, J. P. Dowling, C. M. Bowden, and A. S. Manka, J. Appl. Phys. 83, 2377 (1998).

${ }^{3}$ M. R. Gadsdon, J. Parsons, and J. R. Sambles, J. Opt. Soc. Am. B 26, 734 (2009).

${ }^{4}$ C. García-Meca, J. Hurtado, J. Martí, A. Martínez, W. Dickson, and A. V. Zayats, Phys. Rev. Lett. 106, 067402 (2011).

${ }^{5}$ R. Ulrich, Infrared Phys. Technol. 7, 37 (1967).

${ }^{6}$ R. Ulrich, Infrared Phys. Technol. 7, 65 (1967).

${ }^{7}$ J. Carbonell, C. Croenne, F. Garet, E. Lheurette, J. L. Coutaz, and D. Lippens, J. Appl. Phys. 108, 014907 (2010).

${ }^{8}$ M. Choi, S. H. Lee, Y. Kim, S. B. Kang, J. Shin, M. H. Kwak, K.-Y. Kang, Y.-H. Lee, N. Park, and B. Min, Nature (London) 470, 369 (2011).

${ }^{9}$ C. S. R. Kaipa, A. B. Yakovlev, G. W. Hanson, Y. R. Padooru, F. Medina, and F. Mesa, Phys. Rev. B 85, 245407 (2012).

${ }^{10}$ M. Beruete, M. Sorolla, M. Navarro-Cía, F. Falcone, I. Campillo, and V. Lomakin, Opt. Express 15, 1107 (2007).

${ }^{11}$ M. Navarro-Cía, M. Beruete, F. Falcone, J. M. Illescas, I. Campillo, and M. Sorolla, IEEE Trans. Antennas Propag. 59, 2980 (2011).

${ }^{12}$ C. S. R. Kaipa, A. B. Yakovlev, F. Medina, F. Mesa, C. A. M. Butler, and A. P. Hibbins, Opt. Express 18, 13309 (2010).

${ }^{13}$ C. S. R. Kaipa, A. B. Yakovlev, F. Medina, and F. Mesa, J. Appl. Phys. 109, 044901 (2012).

${ }^{14}$ A. Fallahi and J. Perruisseau-Carrier, Phys. Rev. B 86, 195408 (2012).

${ }^{15}$ A. Yu. Nikitin, F. Guinea, and L. Martin-Moreno, Appl. Phys. Lett. 101, 151119 (2012).

${ }^{16}$ J. Bai, X. Zhong, S. Jiang, Y. Huang, and X. Duan, Nat. Nanotechnol. 5, 190 (2010).

${ }^{17}$ L. Ju, B. Geng, J. Horng, C. Girit, M. Martin, Z. Hao, H. A. Bechtel, X. Liang, A. Zetti, Y. Shen, and F. Wang, Nat. Nanotechnol. 6, 630 (2011).

${ }^{18}$ S. Thongrattanasiri, F. H. L. Koppens, and F. J. Garcia de Abajo, Phys. Rev. Lett. 108, 047401 (2012).

${ }^{19}$ P.-Y. Chen and A. Alù, ACS Nano 5, 5855 (2011).

${ }^{20}$ F. Rana, IEEE Trans. Nanotechnol. 7, 91 (2008).
${ }^{21}$ J. A. Kong, Electromagnetic Wave Theory (Wiley, New York, 1986).

${ }^{22}$ S. Bae, H. Kim, Y. Lee, X. Xu, J. S. Park, Y. Zheng, J. Balakrishnan, T. Lei, H. R. Kim, Y. I. Song et al., Nat. Nanotechnol. 5, 574 (2010).

${ }^{23}$ Y. M. Lin, K. A. Jenkins, A. Valdes-Garcia, J. P. Small, D. B. Farmer, and P. Avouris, Nano Lett. 9, 422 (2009).

${ }^{24}$ F. Xia, D. B. Farmer, Y. M. Lin, and P. Avouris, Nano Lett. 10, 715 (2010).

${ }^{25}$ K. I. Bolotin, K. J. Sikes, Z. Jiang, G. Fudenberg, J. Hone, P. Kim, and H. L. Stormer, Solid State Commun. 146, 351 (2008).

${ }^{26}$ D. Mann, A. Javey, J. Kong, Q. Wang, and H. Dai, Nano Lett. 3, 1541 (2003).

${ }^{27}$ A. Vakil and N. Engheta, Science 332, 1291 (2011).

${ }^{28}$ D. R. Anderson, J. Opt. Soc. Am. B 27, 818 (2010).

${ }^{29}$ E. G. Mishchenko, A. V. Shytov, and P. G. Silvestrov, Phys. Rev. Lett. 104, 156806 (2010).

${ }^{30}$ G. W. Hanson, J. Appl. Phys. 104, 084314 (2008).

${ }^{31}$ C. Xu, H. Li, and K. Banerjee, IEEE Trans. Electron Devices 56, 1567 (2009).

${ }^{32}$ G. Deligeorgis, M. Dragoman, D. Neculoiu, D. Dragoman, G. Konstantinidis, A. Cismaru, and R. Plana, Appl. Phys. Lett. 95, 073107 (2009).

${ }^{33}$ V. V. Popov, T. Y. Bagaeva, T. Otsuji, and V. Ryzhii, Phys. Rev. B 81, 073404 (2010).

${ }^{34}$ M. Dragoman, D. Neculoiu, A. Cismaru, A. A. Muller, G. Deligeorgis, G. Konstantinidis, D. Dragoman, and R. Plana, Appl. Phys. Lett. 99, 033112 (2011).

${ }^{35}$ V. P. Gusynin, S. G. Sharapov, and J. P. Carbotte, J. Phys.: Condens. Matter 19, 026222 (2007).

${ }^{36}$ G. W. Hanson, J. Appl. Phys. 103, 064302 (2008).

${ }^{37}$ HFSS: High Frequency Structure Simulator based on the Finite Element Method, ver. 12.0, Ansoft Corporation [http://www.ansoft.com].

${ }^{38}$ Z. Q. Li, E. A. Henriksen, Z. Jiang, Z. Hao, M. C. Martin, P. Kim, H. L. Stormer, and D. N. Basov, Nat. Phys. 4, 532 (2008).

${ }^{39}$ C. Lee, J. Y. Kim, S. Bae, K. S. Kim, B. H. Hong, and E. J. Choi, Appl. Phys. Lett. 98, 071905 (2011)

${ }^{40}$ J. Y. Kim, C. Lee, S. Bae, K. S. Kim, B. H. Hong, and E. J. Choi, Appl. Phys. Lett. 98, 201907 (2011). 
${ }^{41}$ W. Steinhögl, G. Schindler, G. Steinlesberger, M. Traving, and M. Engelhardt, J. Appl. Phys. 97, 023706 (2005).

${ }^{42} \mathrm{~S}$. Tretyakov, Analytical Modeling in Applied Electromagnetics (Artech House, Norwood, MA, 2003).

${ }^{43}$ O. Luukkonen, C. Simovski, G. Grant, G. Goussetis, D. Lioubtchenko, A. Raisanen, and S. Tretyakov, IEEE Trans. Antennas Propag. 56, 1624 (2008).

${ }^{44}$ A. B. Yakovlev, Y. R. Padooru, G. W. Hanson, A. Mafi, and S. Karbasi, IEEE Trans. Microwave Theory Tech. 59, 527 (2011).

${ }^{45}$ Y. R. Padooru, A. B. Yakovlev, C. S. R. Kaipa, G. W. Hanson, F. Medina, F. Mesa, and A. W. Glisson, IEEE Trans. Antennas Propag. 60, 5727 (2012).
${ }^{46}$ Y. R. Padooru, A. B. Yakovlev, C. S. R. Kaipa, F. Medina, and F. Mesa, Phys. Rev. B 84, 035108 (2011).

${ }^{47}$ D. M. Pozar, Microwave Engineering, 3rd ed. (Wiley, New York, 2004).

${ }^{48}$ P. B. Johnson and R. W. Christy, Phys. Rev. B 6, 4370 (1972).

${ }^{49}$ A. Vial and T. Laroche, J. Phys. D: Appl. Phys. 40, 7152 (2007).

${ }^{50}$ Y. R. Padooru, A. B. Yakovlev, P.-Y. Chen, and A. Alù, J. Appl. Phys. 112, 034907 (2012).

${ }^{51}$ Y. R. Padooru, A. B. Yakovlev, P.-Y. Chen, and A. Alù, J. Appl. Phys. 112, 104902 (2012). 